\title{
Pulse synthesis in the single-cycle regime from independent mode-locked lasers using attosecond-precision feedback
}

\author{
J. A. Cox, ${ }^{1, *}$ W. P. Putnam, ${ }^{1}$ A. Sell,,${ }^{1,3}$ A. Leitenstorfer, ${ }^{3}$ and F. X. Kärtner ${ }^{1,2}$ \\ ${ }^{1}$ Department of Electrical Engineering and Computer Science, and Research Laboratory of Electronics, \\ Massachusetts Institute of Technology, 77 Massachusetts Avenue, Cambridge, Massachusetts 02139, USA \\ ${ }^{2}$ Center for Free-Electron Laser Science-DESY, and Department of Physics, Hamburg University, Hamburg, Germany \\ ${ }^{3}$ Department of Physics and Center for Applied Photonics, University of Konstanz, 78457 Konstanz, Germany \\ *Corresponding author: joncox@alum.mit.edu
}

Received April 30, 2012; revised July 12, 2012; accepted July 12, 2012;

posted July 12, 2012 (Doc. ID 167370); published August 23, 2012

\begin{abstract}
We report the synthesis of a nearly single-cycle (3.7 fs), ultrafast optical pulse train at $78 \mathrm{MHz}$ from the coherent combination of a passively mode-locked Ti:sapphire laser (6 fs pulses) and a fiber supercontinuum (1-1.4 $\mu \mathrm{m}$, with $8 \mathrm{fs}$ pulses). The coherent combination is achieved via orthogonal, attosecond-precision synchronization of both pulse envelope timing and carrier envelope phase using balanced optical cross-correlation and balanced homodyne detection, respectively. The resulting pulse envelope, which is only 1.1 optical cycles in duration, is retrieved with two-dimensional spectral shearing interferometry (2DSI). To our knowledge, this work represents the first stable synthesis of few-cycle pulses from independent laser sources. (c) 2012 Optical Society of America

OCIS codes: $140.7090,270.2500,140.3425$.
\end{abstract}

Coherent synthesis of independent lasers has been of considerable interest as a means of overcoming the bandwidth limitations of individual lasers. While much work has focused on coherent beam combining of continuous wave lasers for power scaling [1] , coherent synthesis of few-cycle laser sources has recently received great interest [2-6] . As such sources can produce pulses as short as a single-cycle of the carrier wave, they have been pursued for the study of phase sensitive nonlinear optics, including optically driven field emission [7] , carrier wave Rabi flopping []ㅡ, and high harmonic generation []․

The coherent synthesis from independent, few-cycle ultrafast lasers presents fascinating challenges. It requires attosecond-precision measurement and control of the technical and quantum noise of uncorrelated optical oscillators across more than $200 \mathrm{THz}$ of bandwidth to the subcycle level, such that one coherent frequency comb is formed. To date, progress toward single-cycle optical pulse synthesis has relied on coherent combination of multibranch, nonlinear spectral generation from a single master oscillator. Such an approach renders the high-speed fluctuations in carrier phase and pulse timing between spectral branches as common mode $[5,6]$. However, a method for the coherent synthesis of independent lasers serves as the most flexible approach for singlecycle frequency comb synthesis, allowing the spectrum to be tailored for the scientific application of interest. In contrast, schemes based on nonlinear spectral generation from a single oscillator are limited by the nonlinear broadening and compression mechanisms, and often result in spectral gaps in the output $[\underline{5}, 6]$.

However, attosecond-precision synchronization between uncorrelated few-cycle sources has proven challenging due to the requirement for orthogonal, high-speed detection and control of both carrier envelope phase (CEP) and pulse timing with long-term stability [3,, 8$]$. In this Letter, we report the successful culmination of a decade of research toward the coherent synthesis of nearly single-cycle pulses from independent, few-cycle sources with long-term stability.

An illustration of the system is given in Fig. 1. The laser oscillators consist of a Kerr-lens mode-locked (KLM) Ti:sapphire laser [9] and a fiber supercontinuum source [10] at $78 \mathrm{MHz}$ pulse repetition rate, producing 6 and $8 \mathrm{fs}$ pulses, respectively. The pulses are combined on a $50 \%$ broad-band beam splitter (BBBS) with flat group delay (GD) and transmission from 600 to $1500 \mathrm{~nm}$ []] .

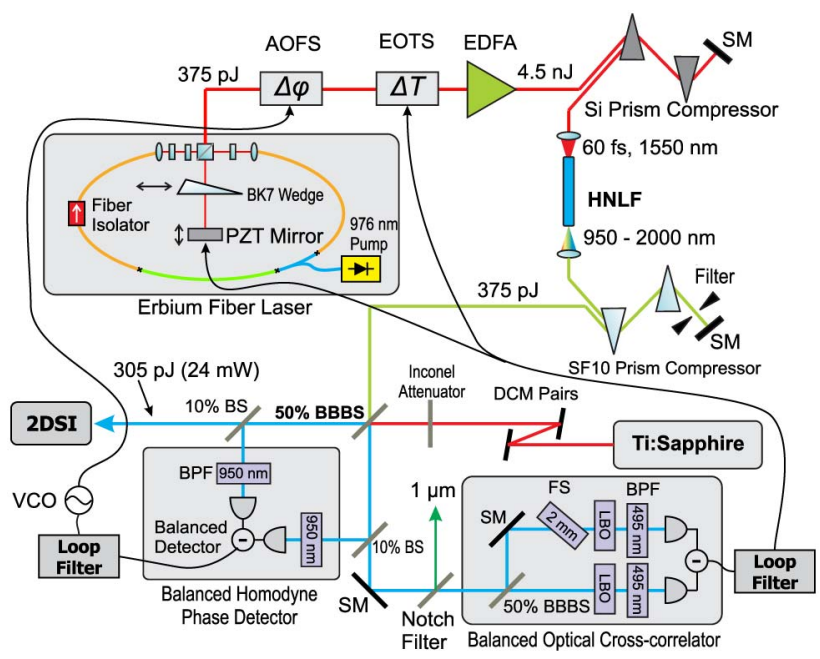

Fig. 1. (Color online) Schematic of system which combines fiber super-continuum and Ti:sapphire pulses on the 50\% BBBS. Pulse envelope timing error is detected by the balanced optical cross-correlator and corrected by feedback upon the piezo mirror and EOTS. CEP error is corrected by the BK7 glass wedge (slow feedback) and the AOFS (fast feedback). In the above: AOFS, acousto-optic frequency shifter; EOTS, electro-optic timing shifter; SM, silver mirror; BS, beam splitter; BBBS, broad band beam splitter; FS, fused silica; BPF, band-pass filter; VCO, voltage controlled oscillator; DCM, double chirped mirrors; 2DSI, two-dimensional spectral shearing interferometer; EDFA, erbium doped fiber amplifier. 
The output of the Ti:sapphire master oscillator, spanning 700-1000 nm, is compressed with double chirped mirrors to $6 \mathrm{fs}$, close to the transform limited duration. Since the Ti:sapphire laser is known to exhibit exceptionally low high-frequency timing jitter at the few attosecond level [11], all feedback is performed upon the fiber supercontinuum source to lock it to the more stable Ti:sapphire laser.

The fiber supercontinuum source is driven by a stretched pulse, passively mode-locked, erbium fiber laser [12]. The timing jitter of this laser was previously determined to be $2.6 \mathrm{fs}$ rms from $10 \mathrm{kHz}$ to the Nyquist frequency [13], by measuring the jitter between two nearly identical fiber lasers. Furthermore, we confirm these earlier measurements by reporting the first absolute jitter measurement of a passively mode-locked fiber laser by measuring against the negligible noise of the Ti:sapphire laser above $10 \mathrm{kHz}$.

The fiber laser pulses are amplified to $4.5 \mathrm{~nJ}$ in an erbium doped fiber amplifier (EDFA) and then compressed to $60 \mathrm{fs}$ duration with a silicon prism pair. Octave spanning supercontinuum is subsequently generated in a Ge-doped, small core highly nonlinear fiber (HNLF) as described in [10]. The short wavelength portion of this supercontinuum (below $1.4 \mu \mathrm{m}$ ) is filtered with a knife edge and compressed to 8 fs duration by a pair of SF10 prisms. The remaining output power is 25 to $30 \mathrm{~mW}$.

Half of the light combined on the BBBS is coupled into a balanced optical cross-correlator (BOC) for timing synchronization [4,14-16]. Critical alignment of the beams is performed with a pair of apertures separated by $2.5 \mathrm{~m}$, while spatial mode matching is achieved via adjustment of the Ti:sapphire cavity. Curved mirrors, instead of lenses, are used for focusing into the lithium triborate (LBO) crystals to avoid spatiotemporal effects that would cause the BOC to lock the pulses with a 500 fs offset. Also, a $1 \mu \mathrm{m}$ notch filter suppresses second harmonic generation in the LBO crystals of the BOC, which would otherwise corrupt the timing signal at $495 \mathrm{~nm}$. As shown in Fig. 2(a), feedback upon the fiber laser's intracavity piezo-actuated mirror results in $2.2 \mathrm{fs}$ rms jitter [10 kHz, $1 \mathrm{MHz}$ ], in good agreement with previously reported results for a pair of fiber lasers [13]. Further reduction of timing jitter to $250 \mathrm{fs}$ rms [ $1 \mathrm{kHz}, 1 \mathrm{MHz}$, or well below the single-cycle level, is obtained via fast feedback upon a waveguide lithium niobate electro-optic phase (timing) shifter, or EOTS, external to the fiber laser cavity, providing a few femtosecond locking range. Fortunately, the EOTS imparts minimal CEP shift since the bulk of the timing jitter is at low frequencies (which is removed by the fast CEP feedback), and because the phase and GD are to within $4 \%$ of each other for lithium niobate.

After obtaining a tight timing lock, the CEP offset frequency between the fiber laser and Ti:sapphire frequency comb is detected at $950 \mathrm{~nm}$ with a balanced homodyne detector. The CEP slip is coarsely adjusted by varying the insertion of a BK7 glass wedge in the fiber laser. Then, fast feedback upon a low-noise, voltage controlled oscillator (VCO) at $175 \mathrm{MHz}$ is used to drive an acousto-optic frequency shifter (AOFS) and lock the relative CEP slip to zero. The AOFS achieves an $f_{\text {CEO }}$ shift of $\pm 5 \mathrm{MHz}$ without resorting to a double-pass configuration to cancel angular walk-off. Moreover, since the
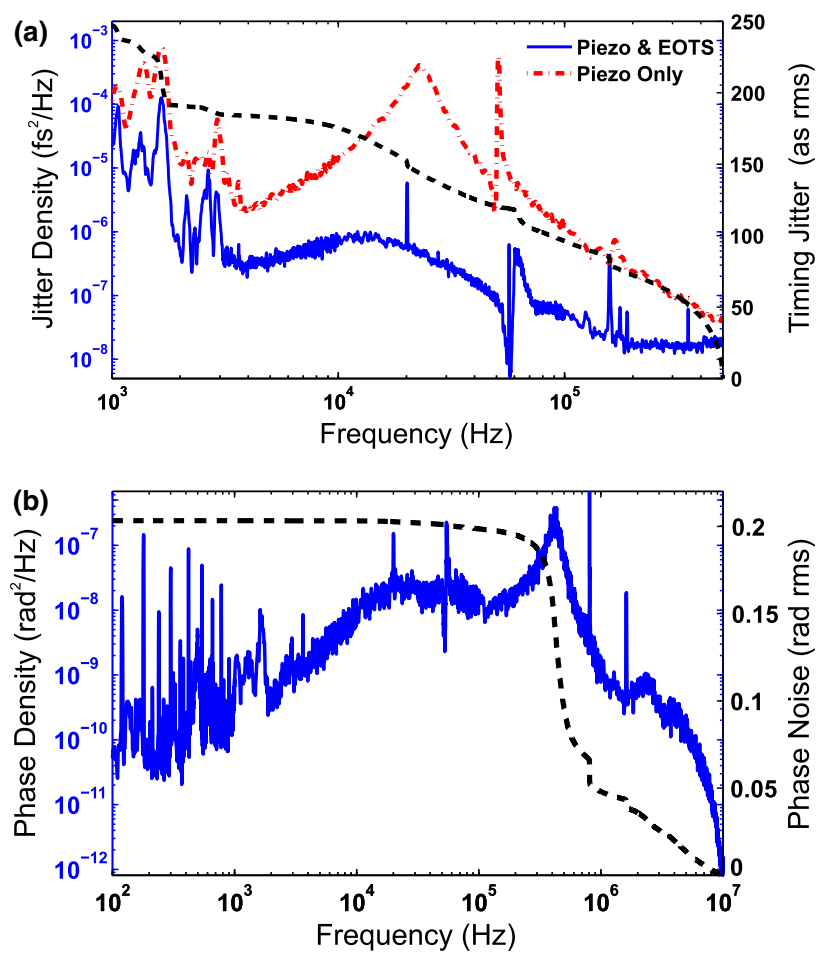

Fig. 2. (Color online) (a) Timing jitter spectral density (JSD) and integrated timing jitter (ITJ) between fiber source and Ti:sapphire laser, as measured at the balanced optical crosscorrelator. JSD after piezo mirror lock (dash-dotted red) yields 2.2 fs rms ITJ. Enabling the EOTS reduces the JSD (blue) and yields only 250 as rms ITJ (dashed black). (b) Residual carrier envelope phase (CEP) noise, as measured at the balanced homodyne detector. CEP is locked to zero offset, yielding $220 \mathrm{mrad}$ rms noise [DC, $5 \mathrm{MHz}$ ].

narrow band optical (60 nm) output of the fiber laser is coupled into the AOFS before the EDFA and HNLF, diffractive broadening is eliminated [17]. Equally as important, feedback CEP stabilization eliminates the slow drift associated with feed forward. As a result, long-term stable synchronization within $200 \mathrm{mrad} \mathrm{rms}$ is achieved with $450 \mathrm{kHz}$ closed loop bandwidth, Fig. 2(b). CEP shifting via pump power modulation is not possible since a nonlinear timing shift of at least $0.1 \mathrm{fs} /$ roundtrip results, breaking the timing lock [18].

The BOC and balanced homodyne detectors provide attosecond resolution measurement of timing and carrier phase difference. Precise measurement of the resulting pulse envelope is necessary to confirm proper temporal overlap and pulse compression. Accurately characterizing a nearly single-cycle pulse with conventional techniques is challenging because increasingly complex schemes are required to reach single-cycle resolution $[5,19]$. On the other hand, two-dimensional spectral shearing interferometry (2DSI) has been successfully adopted for the measurement of few-cycle pulses, since it is largely insensitive to bandwidth limitations and provides a direct measurement of spectral GD without ambiguities [6,20].

We developed a 2DSI apparatus for measurement from 650 to $1400 \mathrm{~nm}$ incorporating a $30 \mu \mathrm{m}$ thick, Type-II phase matched BBO crystal. An interferogram of the synchronized output, Fig. $\underline{3}$, reveals spectrally flat GD over the entire bandwidth, with the exception of the portion near 


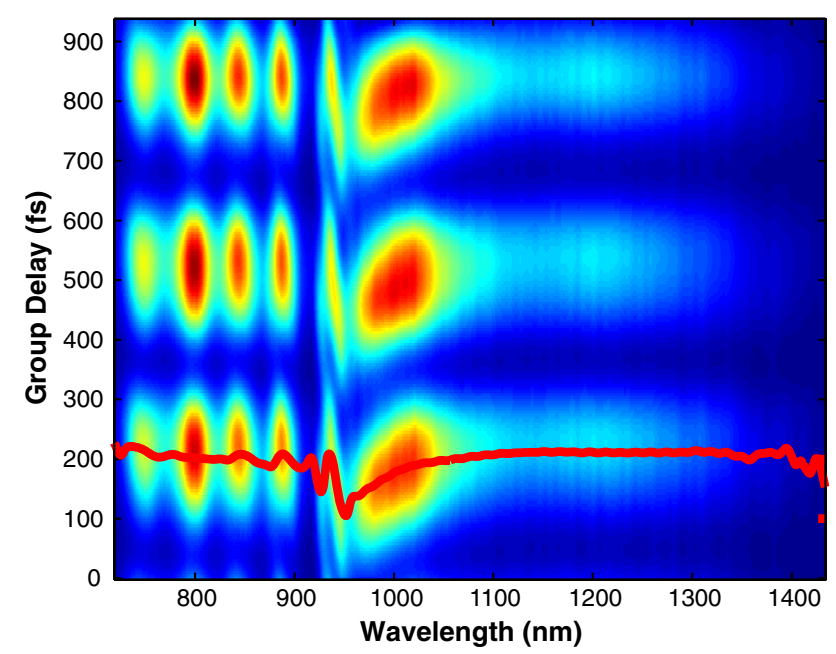

Fig. 3. (Color online) 2DSI after laser synchronization. Spectral group delay (GD), red line, is directly retrieved from the interference fringes.

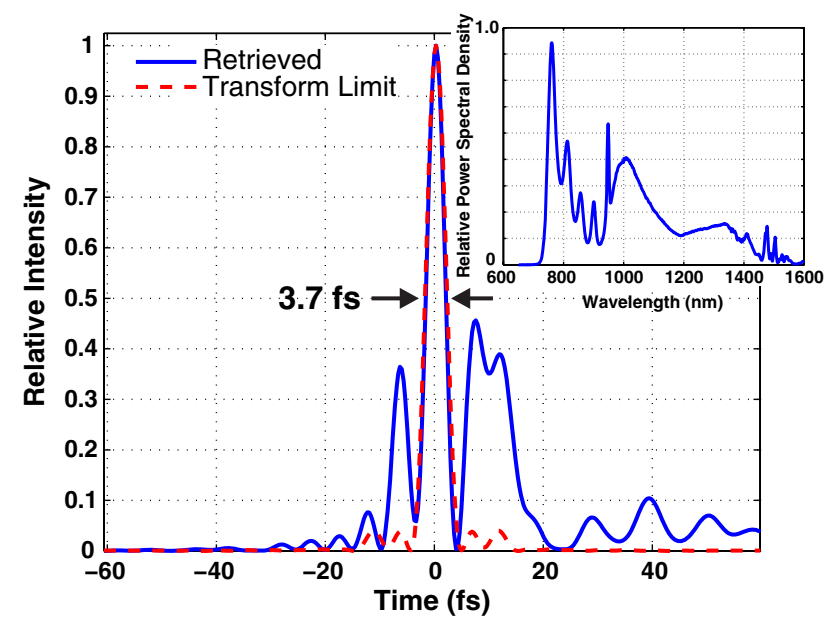

Fig. 4. (Color online) Retrieved pulse intensity (blue) from 2DSI. Pulse duration is $3.7 \mathrm{fs}$ full width half-maximum or 1.1 cycles at the carrier wavelength of $1 \mu \mathrm{m}$. The transformed limited spectrum is also shown (dotted red). Inset: optical power spectrum density for the pulse.

$950 \mathrm{~nm}$, where the third order dispersion of the SF10 prisms and double chirped mirrors increases. The spectral GD retrieved from the 2DSI is combined with a measurement of the fundamental optical power spectral density to yield the complex spectrum. As shown in Fig. $\underline{4}$, the central peak of the pulse coincides with the transform limited spectrum, and has a full width halfmaximum intensity duration of only $3.7 \mathrm{fs}$, or 1.1 optical cycles at $1 \mu \mathrm{m}$. In addition, $44 \%$ of the pulse energy is contained in the central peak, as compared to the two adjacent satellite pulses.

In conclusion, we have reported a method for coherent synthesis of independent, few-cycle ultrafast lasers resulting in nearly single-cycle pulses. Attosecondprecision balanced optical detection of the pulse envelope and carrier phase provide long-term stability of at least several minutes in an uncontrolled laboratory environment. To achieve subcycle, orthogonal control of pulse timing and carrier phase, novel EOTS and AOFS feedback is implemented to overcome the limitations of mechanical and pump power based actuators.

The authors acknowledge support by Department of Energy grant DE-SC0005262 and Air Force Office of Scientific Research grant FA9550-10-1-0063. J. A. Cox acknowledges partial support from a Ford Foundation predoctoral fellowship, W. P. Putnam from a NSF graduate fellowship, and A. Sell from a Feodor-Lynen fellowship of the Alexander von Humboldt Foundation.

\section{References}

1. S. J. Augst, T. Y. Fan, and A. Sanchez, Opt. Lett. 29, 474 (2004).

2. D. J. Jones, J. K. Ranka, A. Stentz, R. S. Windeler, J. L. Hall, and S. T. Cundiff, Science 288, 635 (2000).

3. R. K. Shelton, L. Ma, H. C. Kapteyn, M. M. Murnane, J. L. Hall, and J. Ye, Science 293, 1286 (2001).

4. T. R. Schibli, J. Kim, O. Kuzucu, J. T. Gopinath, S. N. Tandon, G. S. Petrich, L. A. Kolodziejski, J. G. Fujimoto, E. P. Ippen, and F. X. Kaertner, Opt. Lett. 28, 947 (2003).

5. G. Krauss, S. Lohss, T. Hanke, A. Sell, S. Eggert, R. Huber, and A. Leitenstorfer, Nature Photon. 4, 33 (2009).

6. S.-W. Huang, G. Cirmi, J. Moses, K.-H. Hong, S. Bhardwaj, J. R. Birge, L.-J. Chen, E. Li, B. J. Eggleton, G. Cerullo, and F. X. Kärtner, Nature Photon. 5, 475 (2011).

7. P. Hommelhoff, Y. Sortais, A. Aghajani-Talesh, and M. A. Kasevich, Phys. Rev. Lett. 96, 077401 (2006).

8. T. R. Schibli, O. Kuzucu, J.-W. Kim, E. P. Ippen, J. G. Fujimoto, F. X. Kaertner, V. Scheuer, and G. Angelow, IEEE J. Sel. Top. Quantum Electron. 9, 990 (2003).

9. L.-J. Chen, M. Y. Sander, and F. X. Kärtner, Opt. Lett. 35, 2916 (2010).

10. A. Sell, G. Krauss, R. Scheu, R. Huber, and A. Leitenstorfer, Opt. Express 17, 1070 (2009).

11. A. J. Benedick, J. G. Fujimoto, and F. X. Kärtner, Nature Photon. 6, 97 (2012).

12. H. A. Haus, K. Tamura, L. E. Nelson, and E. P. Ippen, IEEE J. Quantum Electron. 31, 591 (1995).

13. J. A. Cox, A. H. Nejadmalayeri, J. Kim, and F. X. Kärtner, Opt. Lett. 35, 3522 (2010).

14. J. Kim, J. Chen, Z. Zhang, F. N. C. Wong, F. X. Kärtner, F. Loehl, and H. Schlarb, Opt. Lett. 32, 1044 (2007).

15. J. Kim, J. Chen, J. Cox, and F. X. Kärtner, Opt. Lett. 32, 3519 (2007).

16. J. Kim, J. A. Cox, J. Chen, and F. X. Kartner, Nature Photon. 2, 733 (2008).

17. S. Koke, C. Grebing, H. Frei, A. Anderson, A. Assion, and G. Steinmeyer, Nature Photon. 4, 462 (2010).

18. M. Y. Sander, E. P. Ippen, and F. X. Kärtner, Opt. Express 18, 4948 (2010).

19. A. Baltuska, M. S. Pshenichnikov, and D. A. Wiersma, IEEE J. Quantum Electron. 35, 459 (1999).

20. J. R. Birge, R. Ell, and F. X. Kärtner, Opt. Lett. 31, 2063 (2006). 